\title{
Stars in the sky: neurocysticercosis
}

\author{
Sonali Kaushal · Alka Sharma • Bakshish Singh • \\ Vivek Kumar • Vishal Sharma
}

Received: 12 August 2011/Accepted: 28 November 2011/Published online: 11 December 2011

(C) SIMI 2011

A 40-year-old man presented to the emergency department (ED) with complaints of headache for the prior 2 days followed by multiple episodes of generalised tonic clonic seizures resulting in status epilepticus. The seizures were controlled with intravenous diazepam followed by an infusion of phenytoin. The work-up for

\section{S. Kaushal}

Department of Anaesthesiology,

Government Medical College and Hospital,

Chandigarh, India

e-mail: sonalikaushal33@gmail.com

\section{A. Sharma}

Department of Medicine,

Government Medical College and Hospital,

Chandigarh, India

e-mail: alka118@gmail.com

\section{B. Singh}

Department of Nephrology,

Satguru Partap Singh Apollo Hospital,

Ludhiana, India

e-mail: bakshish_bsingh@yahoo.co.in

\section{Kumar}

Department of Nephrology,

Postgraduate Institute of Medical

Education and Research,

Chandigarh, India

e-mail: enigma165@yahoo.co.in

V. Sharma $(\bowtie)$

Department of Gastroenterology,

Postgraduate Institute of Medical

Education and Research,

Chandigarh, India

e-mail: docvishalsharma@gmail.com metabolic parameters including serum electrolytes, random blood sugar, arterial blood gas analysis and liver and kidney function tests was non-contributory. Fundus examination revealed bilateral papilledema. The noncontrast computed tomogram of head revealed multiple calcified lesions across the cerebral cortex akin to a starry sky appearance (Fig. 1). Magnetic resonance imaging of the brain revealed multiple bright lesions on $\mathrm{T} 2$ weighted images (Fig. 2). There was no history of skin swellings or previous episodes of seizures. A diagnosis of neurocysticercosis was therefore considered. The x-ray study of the limbs to look for cysticerci was negative. Serum cysticercus IgG antibodies by ELISA were positive. The patient was initiated on phenytoin, mannitol and prednisolone. The patient improved, and was discharged a week later. He was later administered albendazole for 3 weeks. He is still on phenytoin and remains asymptomatic.

Cysticercosis results from infestation by Cysticercus cellulosae, the larval form of pork tapeworm Taenia solium. Hematogenous dissemination results in various systemic manifestations including neurocysticercosis. Neurocysticercosis usually presents with epilepsy. In the developing world, neurocysticercosis is amongst the commonest cause of epilepsy. Although some authors have described a starry sky pattern as a non-ictal manifestation, others (as in our case,) have described seizures as the presenting manifestation in patients with similar findings on brain imaging $[1,2]$. Therapy in patients presenting with seizures should include, apart from antiepileptics, measures to reduce intracranial tension and perilesional edema. Use of antilarval medications early on should be avoided as this can result in exacerbation of the inflammatory reaction, and contribute to a fatal outcome [3]. 


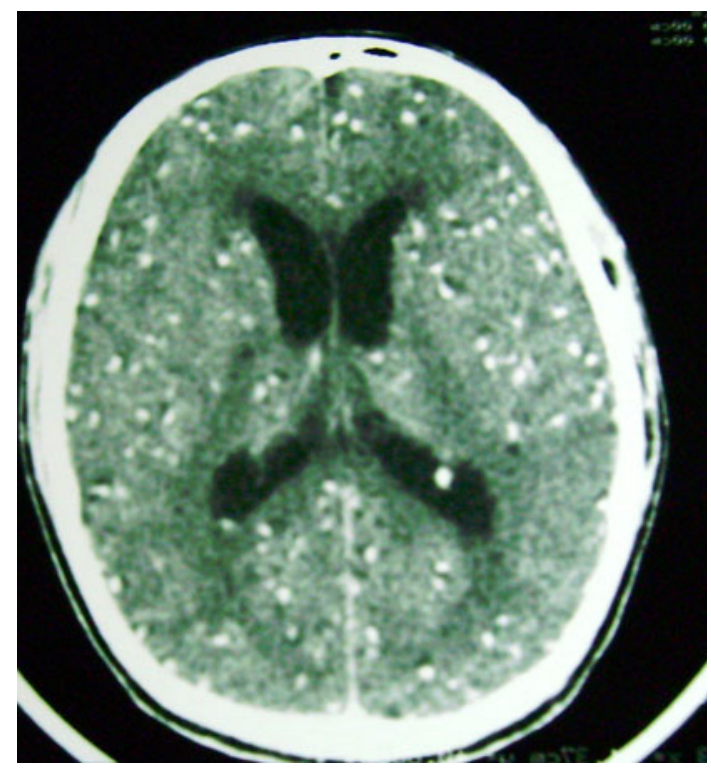

Fig. 1 Computed tomogram of head showing multiple calcified lesions

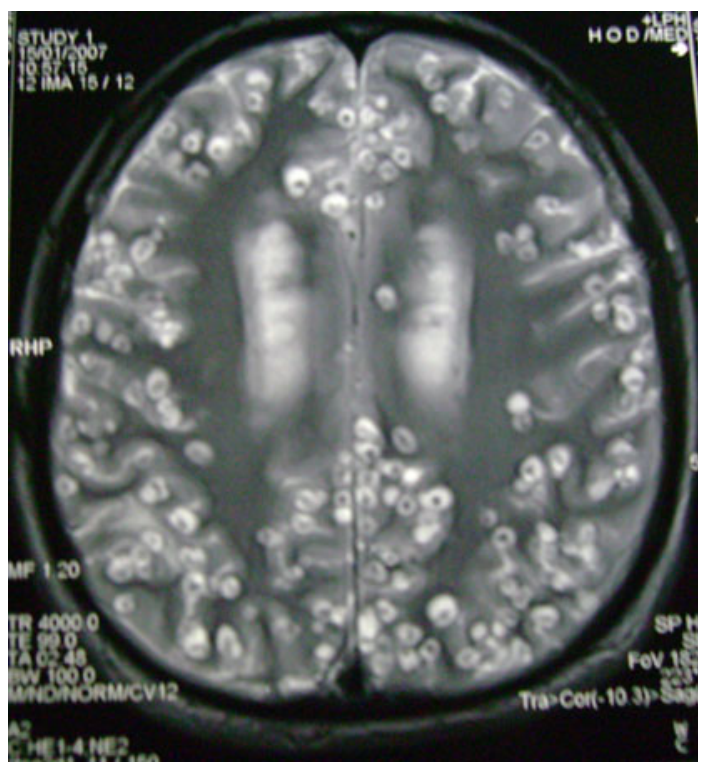

Fig. 2 Magnetic resonance imaging of the brain revealed multiple bright lesions on $\mathrm{T} 2$ weighted images

\section{Conflict of interest None.}

\section{References}

1. Kishore D, Baranwal S, Misra S (2006) Neurocysticercosis causing starry sky appearance-a non-ictal manifestation. J Assoc Phys India 54:464

2. Bhalla A, Sood A, Sachdev A, Varma V (2008) Disseminated cysticercosis: a case report and review of the literature. J Med Case Rep 2:137

3. Das RN (2005) A lesson from a death. BMJ 331:1005 